\title{
Segurança alimentar e nutricional: desenvolvimento de indicadores e experimentação em um município da Bahia, Brasil ${ }^{1}$
}

\section{Food and nutrition security: development of indicators and experimentation in a city of Bahia, Brazil}

Bárbara Eduarda PANELLI-MARTINS ${ }^{2,3}$

Sandra Maria Chaves dos SANTOS 3,4

Ana Marlúcia Oliveira ASSIS

RES U M O

\section{Objetivo}

O objetivo foi propor e aplicar uma metodologia sensível ao fenômeno da segurança alimentar e viável para o monitoramento e a avaliação em municípios de pequeno porte, tendo um município pequeno e pobre como Mutuípe, na Bahia, Brasil, como ilustração.

\section{Métodos}

A partir de pesquisa em várias fontes nacionais e internacionais, da seleção de um conceito guia de segurança alimentar e nutricional e da identificação das suas dimensões, foi elaborado um protocolo com 20 indicadores incluindo variáveis consideradas capazes de expressar a situação no município. 0 protocolo contempla também as premissas que dão inteligibilidade ao modelo, os meios de verificação, os cálculos dos indicadores, os parâmetros e o plano de análise dos resultados.

\section{Resultados}

A aplicação da metodologia no município mostrou-se viável, sendo que a não disponibilidade de dados relevantes para a mensuração da segurança alimentar e nutricional foi um dos principais fatores de constrangimento. No entanto, foi possível identificar que em Mutuípe, considerando as dimensões da disponibilidade, acesso e consumo de alimentos e a utilização biológica de nutrientes, a insegurança alimentar e nutricional é moderada.

\footnotetext{
${ }^{1}$ Artigo elaborado a partir do projeto "Apoio ao desenvolvimento do sistema municipal de segurança alimentar e nutricional de Mutuípe Bahia" - SAN MUTUÍPE. Apoio financeiro: Ministério da Ciência e Tecnologia, Ministério do Desenvolvimento Social e Combate à Fome e Conselho Nacional de Desenvolvimento Científico e Tecnológico, a partir do Edital MCT/MESA/CN Pq/CT-Agronegócio 01/2003 (processo CNPq no 503680/2003-6).

2 Secretaria Estadual de Desenvolvimento Social da Bahia. Salvador, BA, Brasil.

3 Universidade Federal da Bahia, Escola de Nutrição, Programa de Pós-Graduação em Alimentos, N utrição e Saúde. R. Araújo Pinho, 32, Canela, 40110-150, Salvador, BA, Brasil. Correspondência para/Correspondence to: B.E. PANELLI-MARTINS. E-mails: Łbarbara_panelli@yahoo.com.br>; Łpanelli@hotmail.com>.

4 Universidade Federal da Bahia, Escola de Administração, Programa de Pós-Graduação em Administração. Salvador, BA, Brasil.
} 


\section{Conclusão}

O protocolo proposto é operacional e capaz de expressar a situação desejada em municípios pequenos, sendo útil à orientação das políticas locais.

Termos de indexação: Avaliação nutricional. Indicadores. Segurança alimentar e nutricional.

\section{A B S T R A C T}

\section{Objective}

The objective was to propose and apply a methodology that would be sensitive to the phenomenon of food security and viable for monitoring and evaluating small municipalities, using the small, poor city of Mutuípe, in the state of Bahia, Brazil, as an illustration.

\section{Methods}

A protocol consisting of 20 indicators which included variables considered capable of expressing the situation of food security in the municipality was developed based on research from national and international sources, the selection of a guiding framew ork on food and nutrition security, and on the identification of its dimensions. The protocol also contemplates premises that make the model intelligible, means of verification, calculations of the indicators, and the parameters and plan for analysis of the results.

\section{Results}

The application of the methodology in the municipality proved to be viable. One of the main constraining factors was the lack of relevant data for the measurement of food and nutrition security. Nevertheless, it was possible to identify that food and nutrition insecurity in Mutuípe is moderate, taking into consideration the dimensions of food availability, access, consumption, and biological utilization of nutrients.

\section{Conclusion}

The proposed protocol is operational and capable of expressing the desired situation in small municipalities and is useful for guiding local policies.

Indexing terms: Nutrition assessment. Indicators. Food security.

\section{N T R O D U Ç Ã O}

A Segurança Alimentar e Nutricional (SAN) ocupa espaço privilegiado na agenda política brasileira contando, inclusive, com a Lei Orgânica de Segurança Alimentar e Nutricional (LOSAN) sancionada em 2006, que instituiu o Sistema de Segurança Alimentar e Nutricional (SISAN). Tal sistema contempla a atuação das três esferas de governo e da sociedade civil na formulação e programação de políticas, programas e ações para garantia do direito humano à alimentação adequada, cabendo-Ihe ainda monitorar e avaliar o que vem sendo feito nessa área ${ }^{1}$.

$\mathrm{Na}$ esteira desse processo interessa aperfeiçoar os métodos para avaliação dos níveis de SAN de populações, como forma de melhor dirigir as ações e para a avaliação de impactos. Desde 2000, a Organização para Agricultura e Ali- mentação das Nações Unidas (FAO) vem investindo na proposição de indicadores básicos para o monitoramento e a avaliação da situação de segurança alimentar dos países membros². No Brasil, como signatário dos planos de ação pactuados nas Cúpulas Mundiais de Alimentação e, particularmente, por um movimento social e político que construiu um importante espaço para 0 debate e a ação em torno da SAN ${ }^{3-5}$, a preocupação com melhores métodos para avaliar o fenômeno vem se fazendo presente nos debates das Conferências Nacionais de Segurança Alimentar e Nutricional e na LOSAN.

Progressivamente a preocupação com a avaliação da SAN no Brasil alcançou a esfera municipal, tendo em vista a descentralização de políticas sociais. 0 município, assim, passa a ser 0 locus privilegiado para a implementação das ações voltadas à promoção da SAN. 0 município deve, 
portanto, ser capaz de identificar os grupos socialmente vulneráveis ao problema, tanto quanto de acompanhar e avaliar os resultados das ações a eles dirigidas.

No entanto, é reconhecida a limitada ou inexistente experiência de avaliação da intervenção pública em geral e, mais ainda, no plano municipal. A avaliação da SAN não escapa a esta situação, especialmente porque não se conta, ainda, com métodos que possam dar conta de avaliar o fenômeno em toda a sua extensão.

Dessa forma, este artigo apresenta uma metodologia para a construção de um protocolo de indicadores para avaliação da situação de SAN municipal, a partir de dados e informações secundárias. Contempla, também, a análise e a discussão dos resultados obtidos com a aplicação do protocolo na avaliação do problema em um município baiano.

\section{M É TO D O S}

O município de Mutuípe está localizado no Recôncavo Baiano com cerca de 20500 habitantes, dos quais $56,0 \%$ concentram-se na zona rural. A prefeitura municipal é o maior empregador local. Os trabalhadores recebem, em sua maioria, um salário-mínimo e cerca de 17,4\% da população acima de 10 anos é analfabeta. 0 município possui condições precárias de saneamento básico, principalmente na zona rural. Sua economia gira em torno da agricultura familiar ${ }^{6}$.

Em 2000 foi realizado o diagnóstico de saúde e nutrição da população, a partir do qual foram identificados como importantes problemas de saúde e nutrição a anemia em menores de 5 anos, a hipertensão arterial e o sobrepeso/obesidade entre os adultos e idosos; nestes últimos incluise ainda a alteração dos níveis glicêmicos ${ }^{6}$.

Em um segundo momento, foi elaborado e executado o projeto de Apoio ao Desenvolvimento do Sistema Municipal de Segurança Alimentar e Nutricional de Mutuípe (BA), com financiamento do Conselho Nacional de Desenvolvimento Científico e Tecnológico (CNPq) e apoio do executivo municipal. Este projeto contemplou seis subprojetos voltados para várias dimensões do fenômeno da SAN. Um dos subprojetos objetivou desenvolver metodologias para avaliação da SAN, aplicar e avaliar a situação municipal. Este estudo, dessa forma, é um produto desse subprojeto.

\section{A estratégia de avaliação da SAN municipal}

O fenômeno da SAN, por definição, comporta vários aspectos. Se assim é, avaliá-lo exige metodologias capazes de se aproximarem 0 máximo possível das múltiplas facetas do problema em duas dimensões intercomplementares: avaliação de situações de risco e avaliação de intervenções.

Avaliar situações de risco é fundamental para a tomada de decisão em relação à necessidade de intervir, para quem, em que local, de que forma. Trata-se, portanto, de uma tarefa estratégica para evidenciar as situações de insegurança alimentar vividas pela população e para definir prioridades de intervenção. A avaliação de situações de risco é também importante para avaliação do impacto de intervenções, ainda que, nem sempre, possa responder sobre que ações foram mais efetivas e eficazes. Assim, para avaliar o impacto das intervenções podem ser adotados outros métodos.

Em uma breve revisão sobre os métodos a serem adotados para a avaliação da SAN municipal considera-se que os mesmos podem ser classificados em métodos para avaliação da disponibilidade e acesso aos alimentos; métodos baseados no consumo alimentar ${ }^{7,8}$; métodos baseados na Escala de Avaliação Psicométrica9,10 e métodos referidos à situação de saúde e nutrição ${ }^{8}$. Todos esses métodos têm vantagens e desvantagens, particularmente quando se consideram as condições dos municípios em operacionalizá-los. 
68 B.E. PANELLI-M ARTINS et al.

Neste estudo, voltado para o desenvolvimento de uma metodologia de avaliação da SAN que possa ser aplicada de forma sistemática, nas condições técnicas e administrativas dos municípios brasileiros, optou-se pela proposição de um protocolo de indicadores como detalhado na continuidade deste artigo.

\section{RESULTA D OS E DISCUSSÃ O}

\section{A escolha por um protocolo de indicadores}

Um indicador é uma medida que aponta certa condição, característica, atributo ou medida numérica, que permite o registro, a compilação e a análise de dados e informações so bre um evento, tornando possível a mensuração de conceitos mais complexos $^{11-13}$. 0 indicador pode ser representado por um número, por uma relação entre dois eventos ou por uma qualidade ou atributo do fenômeno em observação. Destaca-se ainda que o indicador só seja válido no contexto específico no qual se processa a avaliação.

Para avaliar a SAN, o conceito de indicador adotado se aproxima do adotado pela Organização Pan-Americana da Saúde (OPAS) ${ }^{14}$. Indicadores são, portanto, medidas-síntese que contêm informações relevantes sobre determinados atributos e dimensões do estado de SAN, informando aspectos da realidade ou mudanças em processo, tendo em vista a formulação de políticas públicas, além de esclarecer as mudanças e os determinantes dos diferentes fenômenos.

Desde 1996 a FAO ${ }^{15}$ alerta quanto à necessidade da criação de um protocolo de indicadores básicos relacionados diretamente com a SAN, para ser utilizado como padrão internacional, nacional e/ou local, de forma que seja possível avaliá-la nessas esferas.

Atualmente, para o âmbito local, a FAO disponibiliza um guia municipal que visa a auxiliar os governos à construção do plano de ação. Neste guia está proposto um protocolo simplificado de indicadores divididos em quatro dimensões da SAN, a saber: disponibilidade de alimentos, acesso aos alimentos, consumo de alimentos e utilização biológica de nutrientes ${ }^{16,17}$.

A FAO, porém, admitindo as limitações dos países em desenvolvimento para a produção e atualização das informações pertinentes à elaboração dos indicadores, sugere aos pesquisadores que adotem aqueles que melhor correspondam à sua realidade. Como fator limitante à seleção desses indicadores observa-se a falta de dados relevantes para a maioria dos indicadores enumerados. Além disso, inexiste uma base de dados com periodicidade de atualização impedindo, na maioria dos países em desenvolvimento, que os dados sejam notificados de maneira sistemática ${ }^{18}$.

\section{A construção do protocolo}

\section{Definição do foco da avaliação}

A literatura da área considera que uma avaliação pode ter foco nos resultados, nos processos, ou em ambos. 0 foco nos resultados pressupõe a análise dos impactos gerados a partir de determinadas intervenções ${ }^{19,20}$. No caso da avaliação de um programa em SAN importaria, portanto, verificar se ocorreram mudanças nos níveis de segurança ou insegurança alimentar de uma população. M as, uma avaliação de resultados também se preocupa em reconhecer que fatores atuaram durante a implementação, de forma a obstar ou potencializar o alcance dos objetivos desejados. Um dos maiores desafios metodológicos neste caso está em coletar dados empíricos que permitam considerar o que teria ocorrido com os beneficiários, se o programa não tivesse sido implementado.

Uma avaliação com foco nos processos, por sua vez, volta-se para investigar o funcionamento dos programas, buscando a identidade entre ação e resultado ${ }^{19}$.

No caso desta metodologia proposta, na medida em que foi desenhada de forma a avaliar 
a situação de SAN em um município, pode-se admitir que se aproxime de uma avaliação que pretende dar conta de processos e de resultados. Para tanto, deve ser aplicada antes do início de uma intervenção, conformando o baseline, tanto quanto periodicamente de forma a orientar reajustes nas ações e no monitoramento e ao final da intervenção, avaliando resultados.

Para considerar os resultados desejados tendo como norte um determinado nível de SAN na população, adotou-se o conceito estabelecido pela $\operatorname{LOSAN}^{1}$, a saber:

Art. 3‥ A segurança alimentar e nutricional consiste na realização do direito de todos ao acesso regular e permanente a alimentos de qualidade, em quantidade suficiente, sem comprometer 0 acesso a outras necessidades essenciais, tendo como base práticas alimentares promotoras de saúde que respeitem a diversidade cultural e que sejam ambiental, cultural, econômica e socialmente sustentáveis.

$\mathrm{Na}$ medida em que a complexidade do fenômeno da SAN permite a desagregação conceitual em, pelo menos, quatro dimensões, para a elaboração do protocolo de indicadores foram identificadas e definidas as dimensões, a saber: 1) Disponibilidade dos alimentos, que se refere ao transporte, à produção e à comercialização de alimentos no município que podem limitar a oferta de alimentos à população; 2) Acesso aos alimentos, referindo-se a fatores sociais, econômicos e culturais que interferem no consumo de alimentos; 3) Consumo de alimentos, que inclui aspectos relacionados às condições de saúde e nutrição que reflitam o consumo de alimentos e; 4) Utilização biológica de nutrientes, que se relaciona às condições de acesso a serviços sociais, de saneamento e de saúde que possam limitar a utilização dos nutrientes presente nos alimentos consumidos.

Entende-se que os melhores níveis de SAN de uma população dependem de resultados bons em indicadores de todas as dimensões, sendo que com um protocolo que permita uma leitura de resultados desagregados, pode o executivo municipal reconhecer onde estão concentrados seus problemas e que tipo de ação se faz necessária.

\section{Seleção e definição de indicadores}

Para a seleção foram pesquisados os indicadores existentes nacional e internacionalmente, em um processo de duas etapas: 1) foram selecionados os que melhor atendiam às propriedades desejáveis e 2) entre os primeiros, foram selecionados os que atendiam às características desejáveis.

Segundo Jannuzzij ${ }^{11}$, as propriedades desejáveis para os indicadores são: (a) a relevância social, que justifica e legitima o seu emprego nos processos de análise; (b) a validade de constructo, que corresponde ao grau de proximidade entre 0 conceito e a medida, ou seja, a capacidade de refletir o conceito abstrato ao qual o indicador se propõe a operacionalizar; e (c) a confiabilidade, que é a propriedade relacionada à qualidade do levantamento dos dados usados em seu cômputo.

Jannuzzi ${ }^{11}$ ressalta ainda a necessidade da observância das características desejáveis para indicadores sociais. Além disso, considerou-se as observações do estudo desenvolvido por Machado ${ }^{21}$, que realizou uma comparação entre indicadores para avaliação da pobreza na Bahia. A partir desses estudos, entende-se que a escolha de indicadores e de outros métodos para avaliação da situação de SAN deve contemplar as seguintes características: sensibilidade, que diz respeito à sua capacidade de refletir mudanças significativas se as condições que afetam a dimensão referida se alteram; especificidade, que corresponde à propriedade de refletir alterações estritamente ligadas às mudanças relacionadas à dimensão de interesse; inteligibilidade, que diz respeito à transparência da metodologia de construção do indicador; periodicidade, com a qual o indicador pode ser atualizado; factibilidade em relação ao custo e tempo da sua obtenção; historicidade, que é a propriedade de dispor de séries históricas extensas e comprováveis. 
A seleção de indicadores é uma tarefa delicada, por não existir uma teoria que a oriente de forma objetiva ${ }^{11}$. Para tanto, foi observada a relação recíproca entre indicando e os indicadores propostos, de forma a garantir a validade dos indicadores. Observavam-se também a confiabilidade dos dados para seu cálculo e a inteligibilidade, para que fosse assegurada a sua transparência. Nem sempre os indicadores contemplam todas as propriedades, porém a seleção daqueles propostos esforçou-se por se aproximar das propriedades mais significativas para cada indicador ${ }^{11}$.

Para gerar informações julgadas oportunas e que não dispunham de indicadores previamente estabelecidos, partiu-se para a criação de novos indicadores. Estes foram criados tomando-se como base os mesmos critérios da seleção, de forma a permitir que o município pudesse fazer uso dos dados que habitualmente são coletados pelos diferentes órgãos.

Fator fundamental para a seleção e proposição dos indicadores foi a consideração sobre a fonte dos dados necessários aos cálculos previstos. Após diversos estudos nas diferentes bases de dados disponíveis, optou-se pela base do Instituto Brasileiro de Geografia e Estatística (IBGE) e do Sistema de Informação da Atenção Básica (SIAB), pois eram as que contemplavam maior quantidade das características requeridas para os indicadores, permitindo não apenas o diagnóstico momentâneo, como a avaliação da situação de SAN em momentos anteriores e a garantia dos dados necessários para avaliações futuras.

Conforme consta no Anexo deste artigo, para cada indicador foi formulada uma premissa que visa a estabelecer o elo entre o resultado obtido pelo indicador e o conceito que orienta a avaliação, no caso, o conceito de SAN. Isto se fez estratégia de controle do protocolo de forma que se pudesse, ao final, contar com um elenco mínimo de indicadores capazes de expressar o fenômeno em observação e também para apoiar a análise dos resultados.
De posse dos indicadores propostos, seguiu-se à construção do protocolo no qual são discriminados, para cada indicador, a fórmula ou o critério de sua obtenção, os parâmetros para classificá-los, a pontuação referida a cada resultado, os meios de verificação e as premissas que deverão orientar as análises.

Este protocolo foi apresentado e discutido com especialistas da área e com a liderança e membros do executivo municipal passando por mais ajustes e chegando-se, ao final, a um protocolo com 20 indicadores, apresentados no Anexo. De posse dos dados necessários, procedeuse à aplicação do protocolo para avaliação da SAN em Mutuípe.

Uma vez aplicado o protocolo e considerando-se que avaliar é atribuir valor, importa a uma metodologia de avaliação emitir um julgamento dos dados obtidos, tendo em vista a pactuação em torno de alguns critérios. Essa definição de critérios e valores para julgamento dos resultados obtidos pelos indicadores é, efetivamente, o fundamento que orienta a leitura e a análise dos resultados, dando sentido ao conjunto de informações ${ }^{12,13}$.

No caso deste estudo, o que dá sentido aos resultados são respostas sobre a situação de segurança alimentar e nutricional. Portanto, elaborou-se um sistema de pontuação para traduzir os resultados dos indicadores em diferentes momentos da SAN. Arbitrou-se, então, para cada indicador uma pontuação de 0 a 10, conforme os resultados dos indicadores se aproximassem da garantia da SAN (mais próximo do zero) ou oferecesse risco à insegurança alimentar e nutricional (mais próximo do valor 10).

Importante considerar, também, que alguns indicadores têm resultados dicotômicos. Por exemplo, "existência de cooperativas de pequenos produtores". Assim, o indicador pontua o mínimo para a resposta positiva (0 ponto) e o máximo em caso de resposta negativa (10 pontos). Mas, para a maioria dos indicadores, são possíveis resultados intermediários. Nestes casos foram criados pontos de corte para interpretar os resultados, tendo 
sempre como imagem objetivo a condição daquele resultado somar mais ou menos à SAN.

Ao desagregar a pontuação possível por dimensões observa-se que as dimensões de disponibilidade de alimentos, de acesso aos alimentos, de consumo de alimentos e de utilização biológica de nutrientes podem somar até 70, 40, 30 e 60 pontos, respectivamente. Importa considerar que, no modelo, estas diferenças de pontos possíveis em cada dimensão não pressupõem, necessariamente, uma força maior de uma dimensão em relação à outra, na medida em que se considera que a análise deveria ser integrada. Para garantir peso igual às dimensões, os resultados encontrados para cada dimensão foram aplicados na fórmula abaixo para alcançar os resultados finais da avaliação:

$$
\mathrm{SAN}=[(\mathrm{D} / 7)+(\mathrm{A} / 4)+(\mathrm{C} / 3)+(\mathrm{U} / 6)] / 4, \mathrm{em}
$$

que: nutricional

SAN = Situação de segurança alimentar e

$\mathrm{D}$ = pontuação obtida pela dimensão de disponibilidade de alimentos

A = pontuação obtida pela dimensão de acesso aos alimentos
C = pontuação obtida pela dimensão de consumo de alimentos

$\mathrm{U}=$ pontuação obtida pela dimensão de utilização biológica de nutrientes

De qualquer forma este modelo torna possível, dado um resultado obtido quanto à SAN municipal, reconhecer em quais dimensões estão concentrados os problemas a serem enfrentados.

Para que todos esses números produzidos se tornassem inteligíveis e capazes de emitir um julgamento sobre a situação municipal, construiuse uma escala de classificação da SAN. Como observado na definição dos valores dos indicadores, também aqui se trabalhou com do is pólos: a segurança alimentar e nutricional e a insegurança alimentar e nutricional grave. Entre estes pólos considerou-se a existência de risco à insegurança alimentar e nutricional, a insegurança alimentar e nutricional leve e moderada (Quadro 1).

Para definir que valores obtidos seriam considerados em uma ou outra categoria de SAN adotou-se uma escala de valores, considerando percentuais de alcance da totalidade dos pontos possíveis no plano de indicadores. A escala proposta está apresentada no Quadro 1.

Quadro 1. Escala de valores e categorias para avaliação da segurança alimentar e nutricional municipal com uso de um protocolo de indicadores. Salvador (BA), 2006.

\begin{tabular}{|c|c|c|}
\hline Percentual dos pontos & Categoria & Definição \\
\hline 0 & Segurança alimentar e nutricional & $\begin{array}{l}\text { Representa uma situação ideal em que um conjunto de fatores, } \\
\text { presentes nas dimensões consideradas, atuaria para garantir a reali- } \\
\text { zação plena do conceito de segurança alimentar e nutricional. }\end{array}$ \\
\hline 0,1 a 24,9 & $\begin{array}{l}\text { Risco de insegurança alimentar e } \\
\text { nutricional }\end{array}$ & $\begin{array}{l}\text { Representa uma situação em que alguns fatores, presentes nas } \\
\text { dimensões consideradas, comprometeriam a realização plena do } \\
\text { conceito, mas sem riscos de privação continuada e fome. }\end{array}$ \\
\hline 25 e 49,9 & $\begin{array}{l}\text { Insegurança alimentar e nutricional } \\
\text { leve }\end{array}$ & $\begin{array}{l}\text { Representa uma situação em que alguns fatores, presentes nas } \\
\text { dimensões consideradas comprometeriam a realização plena do con- } \\
\text { ceito, com relativa vulnerabilidade à privação e à fome. }\end{array}$ \\
\hline 50 a 74,9 & $\begin{array}{l}\text { Insegurança alimentar e nutricional } \\
\text { moderada }\end{array}$ & $\begin{array}{l}\text { Representa uma situação em que um maior número de fatores, } \\
\text { presentes nas dimensões consideradas, comprometeria a realização } \\
\text { plena do conceito, caracterizando uma situação de maior } \\
\text { vulnerabilidade à privação e à fome. }\end{array}$ \\
\hline$\geq 75$ & $\begin{array}{l}\text { Insegurança alimentar e nutricional } \\
\text { grave }\end{array}$ & $\begin{array}{l}\text { Representa uma situação em que um maior número de fatores, } \\
\text { presentes nas dimensões consideradas, comprometeria a realização } \\
\text { plena do conceito, caracterizando uma situação de privação e fome. }\end{array}$ \\
\hline
\end{tabular}


É reconhecido que qualquer sistema de classificação é incapaz de dar conta de situações complexas, como é o caso da SAN. O que se intenta, no caso, é permitir uma leitura dos achados, entendendo-se que, progressivamente, com a avaliação dos indicadores e do plano de análise, será possível aperfeiçoar o modelo.

\section{A situação de SAN em Mutuípe}

Um dos princípios orientadores da avaliação da SAN municipal, por meio de indicadores, é a possibilidade de o município contar com uma ferramenta para avaliar o fenômeno, de forma sistemática, com os dados disponíveis em nível local.

Para a avaliação da SAN no município de Mutuípe (BA), fez-se necessária a realização de pesquisas e consultas em diferentes bases de dados que pudessem fornecer os dados e as informações de interesse. Isto é, considerando que se tratou de avaliar a SAN em quatro dimensões, importou ter acesso a dados e informações de diferentes setores.

As condições de acesso aos dados e às informações de várias fontes foram dificuldades importantes para a efetiva avaliação da SAN a partir do protocolo de indicadores.

Foi realizada uma validação qualitativa do protocolo, o que envolveu a apresentação e a discussão dos resultados alcançados por parte de informantes-chaves implicados no fenômeno ${ }^{22,23}$. Isto é, importou saber se aqueles que vivem e atuam no local compreendiam os resultados e estavam de acordo com o que estes sinalizavam quanto às causas e condicionantes do problema.

A metodologia pressupõe outras estratégias de validação a posteriori, por exemplo, a partir de uma análise comparativa com os resultados apresentados pela aplicação da Escala Brasileira de Insegurança Alimentar ${ }^{10}$, também aplicada neste município.

Os resultados encontrados em M utuípe são apresentados no Quadro 2.

Os fatores contribuintes para o resultado quanto a disponibilidade de alimentos foram de diversas ordens. Em termos estruturais destacouse a inexistência de armazéns municipais para estoque de alimentos, na medida em que 0 município deveria possuir capacidade de estocagem de alimentos para abastecer o mercado interno por, pelo menos, dois meses, o que garantiria que problemas na produção/circulação não afetassem o fornecimento de alimentos.

Em termos de organização da produção, a inexistência de cooperativas de pequenos produtores foi também um problema para a disponibilidade de alimentos. Conforme os pressupostos que orientaram a elaboração dos indicadores e dos parâmetros de análise, a existência de cooperativas de pequenos produtores promoveria 0 fortalecimento da produção local, favorecendo o aumento da oferta local de alimentos e 0 aumento da renda das famílias dos pequenos produtores ${ }^{9}$.

Observou-se, também, reduzida proporção de alimentos produzidos localmente e comercializados na feira livre $(34,8 \%)$. Isto é, mais de $60,0 \%$ dos alimentos comercializados na central

Quadro 2. Avaliação da situação de segurança alimentar e nutricional por dimensões em Mutuípe (BA), 2006.

\begin{tabular}{lcccl}
\hline Dimensão & Pontuação máxima possível & Pontuação obtida & Escala de pontos (\%) & Situação de SAN \\
\hline Disponibilidade de alimentos & 70 & 30 & 42,8 & ISAN leve \\
Acesso aos alimentos & 40 & 15 & 37,5 & ISAN leve \\
Consumo de alimentos & 30 & 15 & 50,0 & ISAN moderada \\
Utilização biológica de alimentos & 60 & 40 & 66,7 & ISAN grave \\
\hline Total & 200 & 100 & 50,0 & ISAN moderada \\
\hline
\end{tabular}

SAN: segurança alimentar e nutricional; ISAN: insegurança alimentar e nutricional. 
de abastecimento eram provenientes de outros municípios. Com isto pressupõe-se, de um lado, uma menor aproximação entre o pequeno produtor local e o consumidor, favorecendo a intermediação, que onera o custo dos alimentos. Além disso, pode-se considerar que há dependência externa do município, o que somado à inexistência de armazéns, indica uma situação vulnerável do município quanto a disponibilidade de alimentos.

Quanto ao acesso aos alimentos, um dos fatores que contribuíram para o resultado encontrado foi a renda do responsável pelos domicílios particulares, que está associada à possibilidade de aquisição e utilização de bens e serviços essenciais à manutenção do estado de saúde, em especial à aquisição de alimentos. Em estudo anterior, realizado em Mutuípe por equipe de pesquisadores da Escola de Nutrição da Universidade Federal da Bahia, também se detectou este problema em amostra representativa dos domicílios. Naquele estudo, 85,3\% dos domicílios apresentaram renda de até dois salários-mínimos ${ }^{6}$. Ainda que para a SAN concorram vários fatores, em países como o Brasil, os problemas estruturais de acesso referidos à renda das pessoas é ainda um fator de mais alto poder de determinação de insegurança.

Apresentaram-se como importantes colaboradores para o resultado encontrado, ainda, a escolaridade do responsável pelo domicílio e 0 percentual de famílias chefiadas por mulheres. 0 primeiro, avaliado como anos de estudo, influencia nas chances de obter melhor remuneração no trabalho, assim como na seleção e no preparo dos alimentos, enquanto que famílias chefiadas por mulheres tendem a ter menor renda, o que por pressuposto, compromete o consumo alimentar, a escolha e a preparação dos alimentos.

0 indicador percentual de crianças com baixo peso ao nascer, relacionado com o estado nutricional materno durante a gestação e preditivo do estado de saúde e sobrevida da criança; e 0 indicador percentual de crianças menores de 5 anos com déficit ponderal para idade, que indica um estado de desnutrição atual, refletindo más condições de saúde, renda e educação da família, foram os indicadores responsáveis pelo resultado encontrado na dimensão de consumo alimentar.

Pode-se pressupor que o resultado encontrado para a dimensão de utilização biológica de nutrientes deve-se, principalmente, à baixa cobertura do Programa Saúde da Família no município à época do estudo, na medida em que as equipes do programa atuariam na prevenção e no tratamento de agravos à saúde. A deficiente cobertura do saneamento básico, nela inclusa o acesso à água tratada, a coleta de lixo doméstico e o esgotamento sanitário, colaboram com 0 resultado.

\section{I S C U S SÃ O}

A exemplo de outros estudos de avaliação da Segurança Alimentar e Nutricional, subdividiuse o conceito em dimensões e, para cada uma delas, foi definida a abrangência para a conceituação ${ }^{24}$. Dessa forma foram construídos os indicadores do fenômeno de interesse, garantindose que os indicadores agrupados em cada dimensão refletissem o que realmente se pretendia.

A partir das dimensões de SAN e considerando os indicadores identificados na literatura, fez-se uma primeira sistematização dos mesmos. Com este exercício identificou-se que alguns dos indicadores propostos tendiam a ser muito gerais, ou seja, identificados com macro-estruturas e, portanto, inadequados à realidade municipal. Gradativamente, a partir de leituras e discussões sobre o tema, indicadores mais apropriados foram propostos e localizados nas quatro dimensões adotadas.

Por diversas vezes durante o processo de construção desta proposta fizeram-se necessárias a reavaliação e a discussão dos indicadores, pois haviam indicadores que ficavam na fronteira entre duas dimensões, exigindo uma decisão do pesquisador. Por exemplo: o indicador de renda do responsável pelos domicílios particulares, por vezes foi questionado se pertenceria a dimensão de 
acesso aos alimentos ou na dimensão de consumo de alimentos, tendo ao final sido encaixado na primeira dimensão.

A té o fechamento deste artigo não foi possível encontrar estudos brasileiros para avaliação da SAN municipal, por meio de um protocolo de indicadores. Alguns estudos nacionais fizeram uso de indicadores para avaliação de fenômenos como a desigualdade em saúde ${ }^{25}$ e o perfil de risco para classificação socioeconômica ${ }^{26}$, encontrando dificuldades que se assemelham às identificadas neste trabalho.

Os dados e as informações foram coletados nas bases do Censo Agropecuário e Censo Demográfico-IBGE ${ }^{27}$ e no SIAB, que são as principais fontes citadas pelos estudos brasileiros ou internacionais, por representarem informações de fácil acesso e ampla cobertura.

Um desafio encontrado e sinalizado por outros estudos foi o de selecionar indicadores que fossem capazes de dar conta do fenômeno e constassem nas bases de dados já disponíveis no âmbito nacional e/ou municipal. Conforme indicado, o IBGE foi utilizado como base de dados para os indicadores das dimensões de disponibilidade e de acesso. O SIAB serviu como base para os indicadores de avaliação das dimensões de consumo e de utilização biológica de nutrientes. Porém, em sua base nacional, disponível na rede Internet, foram verificados alguns problemas, como a não disponibilização de dados atualizados para todas as variáveis e a inconsistência de outros.

Outro desafio para esta metodologia foi a definição dos parâmetros para interpretação dos resultados dos indicadores. Isto foi feito em alguns casos tendo em vista definições já aceitas e referenciadas cientificamente ou, em sua ausência, com base na análise dos resultados apresentados nos planos mundial, nacional ou estadual.

Ao tomar como exemplo definição de parâmetros o indicador "percentual de aleitamento materno exclusivo", considerou-se o número de crianças menores de 4 meses nesta condição e se pontuou positivamente para a SAN caso este percentual representasse mais do que $23,4 \%$. Duas escolhas foram realizadas, portanto. A primeira delas quanto ao tempo de duração do aleitamento materno exclusivo. Sem desconhecer a recomendação da Organização Mundial da Saúde ${ }^{28}$ de seis meses, mas tendo em vista a legislação nacional, que garante licença-maternidade por apenas quatro meses, além do fato de que a maioria das mulheres das classes de menor renda trabalha no mercado informal, considerouse mais realista adotar este último como ponto de corte. Assume-se, portanto, em face da realidade nacional e regional, que se a criança está sendo amamentada exclusivamente ao seio até os quatro meses de idade isto soma positivamente para a segurança alimentar e nutricional.

A segunda escolha (relativa aos pontos de corte que definem a condição de adequado/ aceitável/inadequado do indicador percentual de aleitamento materno exclusivo) buscou ter em conta o contexto nacional e local. Isto é, a expectativa é de atingir $100,0 \%$ de aleitamento materno exclusivo até o 4 - mês, no entanto, para o Nordeste, a prevalência encontrada foi de 23,4\% e para o País de $21,6 \%{ }^{29}$. Sendo Mutuípe um município de pequeno porte, nordestino e predominantemente rural, considerou-se que, se naquele contexto fosse alcançada a mesma prevalência nacional, isto configuraria uma situação positiva.

$\mathrm{Na}$ ausência de referências para a definição de parâmetros, em última instância, as autoras arbitraram valores e pontos de corte a partir do conceito de SAN. Há que considerar, também, a flexibilidade dos parâmetros após aplicações seqüenciais, uma vez que o esperado é a modificação da situação. Estas condições do modelo podem consistir em um problema para a reprodutibilidade dos indicadores e para a capacidade do protocolo discriminar diferentes níveis de SAN. A expectativa é a de testar o protocolo em outros municípios, de forma a aperfeiçoar progressivamente os indicadores e seus parâmetros.

Interessou a este estudo analisar ainda quais das propriedades desejáveis para indicadores 
estariam contempladas pelo protocolo proposto ${ }^{11,14}$. Como observado no Quadro 3, buscou-se agregar as questões de interesse em duas grandes dimensões, uma delas voltada para a viabilidade e adequação do uso do método pelo município e a outra contemplando a capacidade do método expressar, de forma clara e objetiva, o problema em foco. Pela análise do método, sistematizada no Quadro 3, pode-se observar que 0 mesmo atende adequadamente às dimensões em análise.

Com base nos resultados apresentados no Quadro 2, classificou-se o município como apresentando insegurança leve para as duas primeiras dimensões, moderada para a dimensão de consumo de alimentos e grave para a dimensão de utilização biológica de nutrientes. Aplicandose a fórmula proposta anteriormente, o município encontra-se em insegurança moderada. A situação identificada no município tem como fatores responsáveis os serviços de saúde, de educação e de saneamento básico deficitários, além da renda, como sugerido por especialistas ${ }^{6,21}$ e observado em outros estudos $9,10,21,26,30$.

Os resultados indicam piores níveis de insegurança alimentar para a dimensão de utilização biológica de nutrientes, seguida pela dimensão de consumo, o que pode justificar-se pela natureza do fenômeno avaliado em cada uma delas. Na primeira dimensão, relacionada às condições de acesso a serviços sociais, de saneamento e de saúde e, na segunda, aos aspectos relacionados às condições de saúde e nutrição que interferem no consumo de alimentos pela família.

A identificação por meio desta avaliação de quais aspectos são responsáveis pela situação

Quadro 3. Matriz analítica do protocolo de indicadores para municípios.

\begin{tabular}{|c|c|c|c|}
\hline \multicolumn{4}{|l|}{ Dimensão: viabilidade e adequação da utilização pelo município } \\
\hline \multirow[t]{2}{*}{ Questões: o método em análise contribui para } & \multirow{2}{*}{ Propriedades relacionadas } & \multicolumn{2}{|c|}{ Resultado } \\
\hline & & Esperado & Encontrado \\
\hline $\begin{array}{l}\text { O fornecimento de tendências sobre o comportamento do problema no } \\
\text { município? }\end{array}$ & Sensibilidade/historicidade & A ou I & Adequado \\
\hline O planejamento de ações em saúde, alimentação e nutrição? & Operacionalidade & A ou I & Adequado \\
\hline Discriminar os grupos vulneráveis ao problema no município? & Focalização & A ou I & Adequado \\
\hline $\begin{array}{l}\text { A compreensão da avaliação (indicadores, variáveis, resultados possíveis) } \\
\text { pelos membros do executivo? }\end{array}$ & Inteligibilidade & A ou I & Adequado \\
\hline $\begin{array}{l}\text { A produção de resultados que possam ser divulgados e compreendidos } \\
\text { pela comunidade em geral? }\end{array}$ & Inteligibilidade & A ou I & Adequado \\
\hline $\begin{array}{l}\text { A realização sistemática da avaliação considerando diferentes recursos ne- } \\
\text { cessários? (financeiros, humanos, materiais) }\end{array}$ & Factibilidade & F ou NF & Factível \\
\hline $\begin{array}{l}\text { Respostas sobre o problema em um tempo adequado às necessidades do } \\
\text { município? }\end{array}$ & Periodicidade & A ou I & Adequado \\
\hline \multicolumn{4}{|c|}{ Dimensão: capacidade de expressar situações de SAN, seus determinantes e resultados gerados pelas intervenções } \\
\hline $\begin{array}{l}\text { Fornecer informações sobre o acesso aos alimentos pelas famílias e sobre } \\
\text { seus determinantes? }\end{array}$ & Sensibilidade & A ou I & Adequado \\
\hline $\begin{array}{l}\text { Evidenciar os determinantes dos problemas de alimentação e nutrição da } \\
\text { população? }\end{array}$ & Especificidade & A ou I & Adequado \\
\hline Informar sobre qualidade da alimentação da população? & Sensibilidade & A ou I & Inadequado \\
\hline Informar sobre aspectos quantitativos da alimentação da população? & Sensibilidade & A ou I & Inadequado \\
\hline Evidenciar mudanças de situações em SAN após intervenções? & Especificidade/sensibilidade & A ou I & Adequado \\
\hline
\end{tabular}

A: adequado; I: inadequado; F: factível; NF: Não factível; SAN: segurança alimentar. 
de SAN pode atuar como norteador para 0 planejamento das políticas e programas municipais das diferentes secretarias, otimizando os recursos disponíveis, a saber: humanos, administrativos e financeiros que, em geral, são escassos em municípios de pequenos porte.

\section{O N C L U SÃ O}

Este estudo apresenta uma metodologia para a construção de um protocolo de indicadores para avaliação da situação de SAN no plano municipal, que, tanto pela viabilidade e adequação da utilização pelo município, quanto pela capacidade de expressar situações de SAN municipal ou intramunicipal, seus determinantes e resultados gerados pelas intervenções, se apresenta como importante instrumento para avaliação no âmbito municipal.

Ao permitir o diagnóstico inicial e a replicação da análise, o protocolo mostra-se instrumental à formulação, ao acompanhamento e à avaliação de intervenções pelo executivo municipal. Apresenta, por outro lado, as condições técnicas e operacionais que corroboram para que o município adote tal metodologia.

A metodologia apresenta, porém, alguns aspectos que devem ser observados quando da adaptação às demais realidades, principalmente no que tange ao estabelecimento dos parâmetros para os indicadores e, em especial, nos casos em que não são encontrados parâmetros previamente estabelecidos na literatura.

Tal método utiliza dados que são coletados periodicamente pelo SIAB e pelo IBGE, o que significa que os mesmos são atualizados regularmente sem a dependência de uma equipe voltada especificamente para este fim, reduzindo a necessidade de recursos humanos e financeiros específicos para a avaliação a partir deste método.

A experiência mostra que o comprometimento do gestor municipal é fundamental para o sucesso da avaliação. Na medida em que a cultura avaliativa ainda é incipiente na administração pública brasileira, o que, por vezes, se verifica é a necessidade de ressaltar, a todo o momento, as vantagens que podem ser obtidas com a avaliação para que se possa contar com a participação efetiva da prefeitura.

Como este método tem como objetivo a aplicação pela própria prefeitura e pelas demais instâncias governamentais, espera-se que a avaliação possa ser mais efetiva, principalmente tendo em vista a aprovação da LOSAN, que, entre outras coisas, destina especial atenção para o desenvolvimento de meios de garantia da SAN e cobra os seus resultados; assim, espera-se a busca por métodos pouco onerosos e de fácil aplicação para o diagnóstico e o acompanhamento das ações.

Portanto, este méto do constitui-se em um importante instrumento para o município, visto permitir a avaliação da situação e a identificação de fatores responsáveis pelos resultados, além de poder ser replicado periodicamente para avaliação dos impactos das ações desenvolvidas e de ser facilmente reconhecido pelos atores sociais responsáveis pela proposição e pelo acompanhamento das ações.

\section{A GRA DECIMENTOS}

A toda equipe do projeto SANMUTUÍPE, Coordenadores de subprojetos: Dalva M aria Fortunato da Nóbrega, Rita de Cássia Ribeiro da Silva, Ryzia de Cássia Vieira Couto. Pesquisadores: Maria da Conceição M onteiro Silva, M ônica Leila Portela de Santana, Nedja Silva Santos, Adenilda Queiroz. Bolsistas de Iniciação Científica, subprojeto avaliação da segurança alimentar e nutricional: Jamille Pereira, Jamile Dacah Fernandes Marchi e a equipe da Prefeitura Municipal de Mutuípe e à comunidade mutuipense.

\section{COLA BORADORES}

B.E. PANELLI-M ARTINS elaborou o projeto específico, realizou trabalho de campo e escreveu o artigo como parte de seu trabalho de conclusão do curso de mestrado. S.M.C. SANTOS coordenou a concepção e a implementação do projeto SANM UTUÍPE, 
realizou trabalho de campo, orientou o trabalho, colaborou na redação do artigo e na revisão final. A.M.O. ASSIS participou da concepção do projeto e da realização do trabalho de campo, da avaliação e da revisão do trabalho final.

\section{RE FERÊ N C IAS}

1. Brasil. Lei Orgânica de Segurança Alimentar e Nutricional. 15 set. 2006 [acesso 2007 fev 2]. Disponível em: <https://www.planalto.gov.br/ consea/static/eventos/LOSAN.pdf>.

2. Comité de Seguridad Alimentaria Mundial. Indicadores básicos propuestos para vigilar la situación de la seguridad alimentaría. Roma; 18-21 de Septiembre 2000 [acesso 2001 out 1]. Disponibile en: <http://www.fao.org/docrep/ meeting/X8228S.htm> .

3. Panelli-Martins BE. Análise de método de avaliação da segurança alimentar e nutricional: uma contribuição à política municipal de SAN. Salvador: UFBA; 2007.

4. Vasconcelos FAG. Combate à fome no Brasil: uma análise histórica de Vargas a Lula. Rev Nutr. 2005; 18(4):439-57.

5. Valente FLS. Do combate à fome à segurança alimentar e nutricional: 0 direito à alimentação adequada. Rev Nutr. 1997; 10(1):20-36.

6. Assis AM O, coordenadora. Diagnóstico de saúde e nutrição da população de Mutuipe. Salvador: EDUFBA; 2002.

7. Rose $D$, Tschirley $D$. Predicting dietary intakes with simple food recall information: a case study from rural Mozambique. Eur J Clin Nutr. 2004; 57(10): 1212-21.

8. Fisberg RM, Slater BM, Dirce ML, Martini LA. Inquéritos alimentares: métodos e bases científicas. Barueri: Manole; 2005.

9. Pérez-Escamilla R. Experiência internacional com a escala de percepção da insegurança alimentar. Cad Estud Desenv Soc Debate. 2005; 2(2):14-25.

10. Segall-Corrêa AM. Segurança alimentar no Brasil: validação de metodologia para acompanhamento e avaliação. Campinas: Unicamp; 2004.

11.Jannuzzi PM. Indicadores sociais no Brasil. Campinas: Alínea; 2001.

12. Guimarães MCL, Santos SMC, Melo C, Sanches Filho A. Avaliação da capacidade de gestão de organizações sociais: uma proposta metodológica em desenvolvimento. Cad Saúde Pública. 2004; 20(6):109-18.
13. Santos SMC, Guimarães MCL, M elo $C$, Sanches Filho A. Subsídios para avaliação da gestão pública: processo de constituição de indicadores para avaliação da capacidade de gestão de organizações sociais. O\&S - Org \& Soc. 2006; 13(13)109-24.

14. Organização Pan-Americana da Saúde. Indicadores básicos de saúde no Brasil: conceitos e aplicações. Rede Interagencial de Informações para a saúde 2002 [acesso em 2007 jan 22]. Disponível em: <http://ww w.tabnet.datasus.gov. br/cgi/ibd2001/ aspectos.pdf $>$.

15. Organización de las Naciones Unidas para la Agricultura y la Alimentación. Declaración de Roma sobre la Seguridad Alimentaria Mundial. Cumbre Mundial sobre la alimentación. 1996 [acceso 2001 out 1]. Disponible en: <http://www.fao.org/docrep/ 003/w3613s/w3613s00.htm>.

16. Olivares S, Garcia C, Salinas G. Relación del município com las famílias que habitan em su espacio territorial. In: Moron C, editor. Guia para la gestión municipal de programas de seguridad alimentaria y nutrición. Santiago del Chile: FAO; 2001. p.8-51.

17. Tácsan L, Rojas Z, López A. Bases para el diseño de un sistema de vigilância alimentaria y nutricional (SISVAN) local. In: Moron C. editor. Guia para la gestión municipal de programas de seguridad alimentaria y nutrición. Santiago del Chile: FAO; 2001. p.53-87.

18. Ribeiro NS, Panelli-M artins BE, Santos SMC. Avaliando a segurança alimentar e nutricional SAN: proposta de um protocolo preliminar de indicadores. Relatório de pesquisa: programa institucional de bolsas de iniciação científica. Salvador: UFBA; 2003.

19. Universidade Estadual de Campinas. Núcleo de Estudos de Políticas Públicas. Programa de Apoio à Gestão Social no Brasil. Projeto: desenho e implantação de estratégia de avaliação. Campinas: Unicamp; 1999.

20. Programa de las Naciones Unidas para el Desarrollo-PNUD. Manual de seguimiento y evaluación de resultados. Oficina de Evaluación del PNUD. New York: ONU; 2002.

21. M achado KM. Uma comparação entre a renda e a abordagem multidimensional da pobreza na Bahia [dissertação]. Bahia: Universidade Federal da Bahia; 2006.

22. Minayo MCS, Assis SG, Souza ER, organizadores. Avaliação por triangulação de métodos. Rio de Janeiro: Fiocruz; 2005. v.1.

23. Guimarães $M C L$, Santos SMC, M elo CS, Sanches Filho A. Avaliação da capacidade de gestão de organizações sociais: uma proposta metodológica 
em desenvolvimento. Cad Saúde Pública. 2004; 20(6):1642-50.

24. Acosta SJ. Métodos de medición de la seguridad alimentaria. Rev Cubana Aliment Nutr. 1995; 9(1):30-5.

25. Drachler ML, Côrtes SMV, Castro JDC, Leite JCC. Proposta de metodologia para selecionar indicadores de desigualdade em saúde visando definir prioridades de políticas públicas no Brasil. Ciênc Saúde Coletiva. 2003; 8(2):461-70.

26. Carvalho MS, Cruz OG, Nobre FF. Perfil de risco: método multivariado de classificação sócioeconômica de micro-áreas urbanas - os setores censitários da região metropolitana do Rio de Janeiro. Cad Saúde Pública. 1997; 13(1):535-45.

27. Instituto Brasileiro de Geografia e Estatística. Censo demográfico brasileiro: Brasil 2000 [acesso
2004 ago 10]. Disponível em: <http://www. ibge.gov.br>.

28. World Health Organization. The optimal duration of exclusive breastfeeding. Report of an expert consultation. Geneva; 2001

29. Brasil. Ministério da Saúde. Prevalência de aleitamento materno exclusivo. [acesso 2005 maio 26]. Disponível em: <http://tabnet.datasus.gov.br/ cgi/idb2001/d20.htm>.

30. Batista Filho M, Rissin A. A transição nutricional no Brasil: tendências regionais e temporais. Cad Saúde Pública. 2003; 19(Supl 1):181-91.

Recebido em: 4/5/2007

Versão final reapresentada em: 8/4/2008 Aprovado em: 24/4/2008 


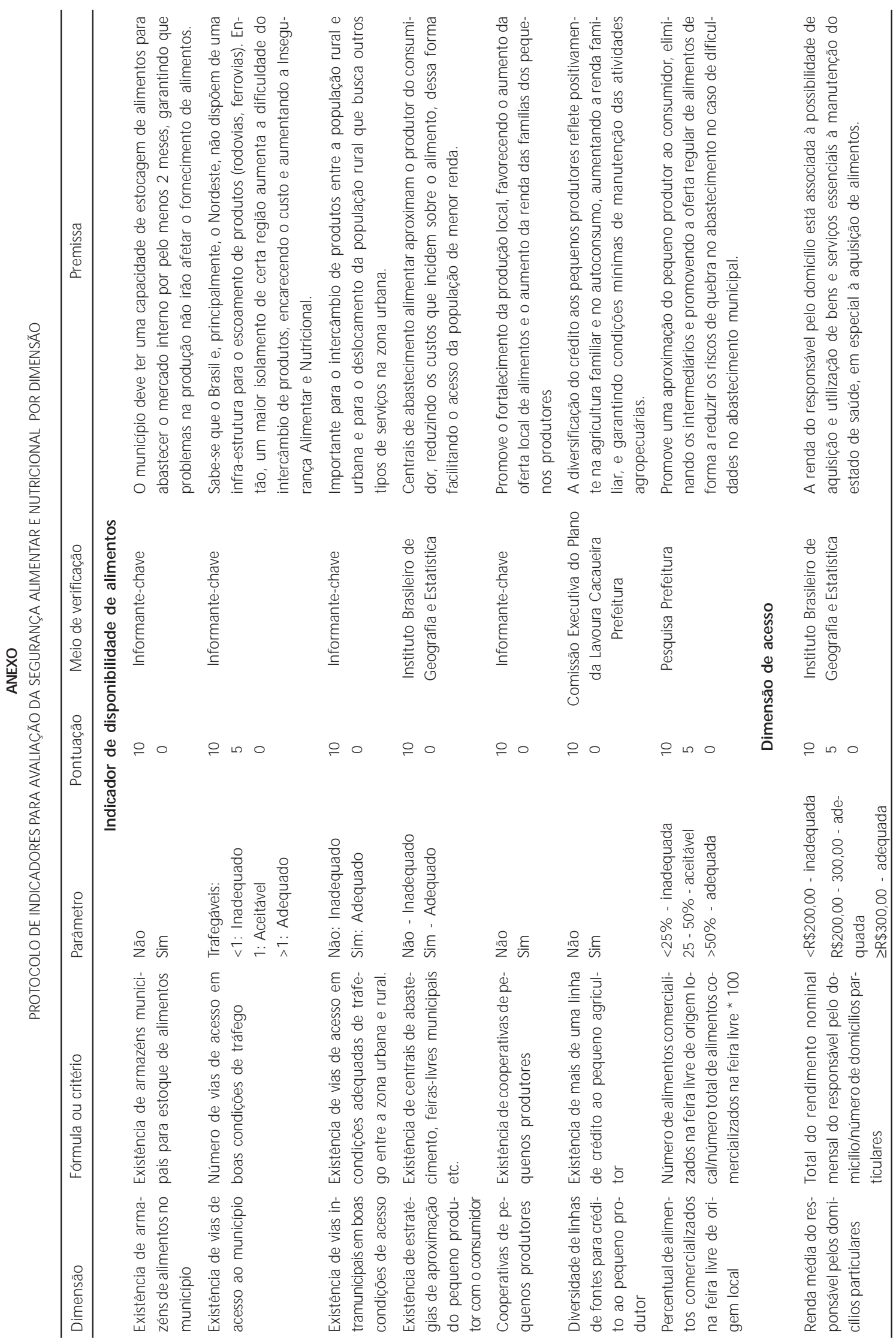


$80 \mid$ B.E. PANELLL-MARTINS et al.

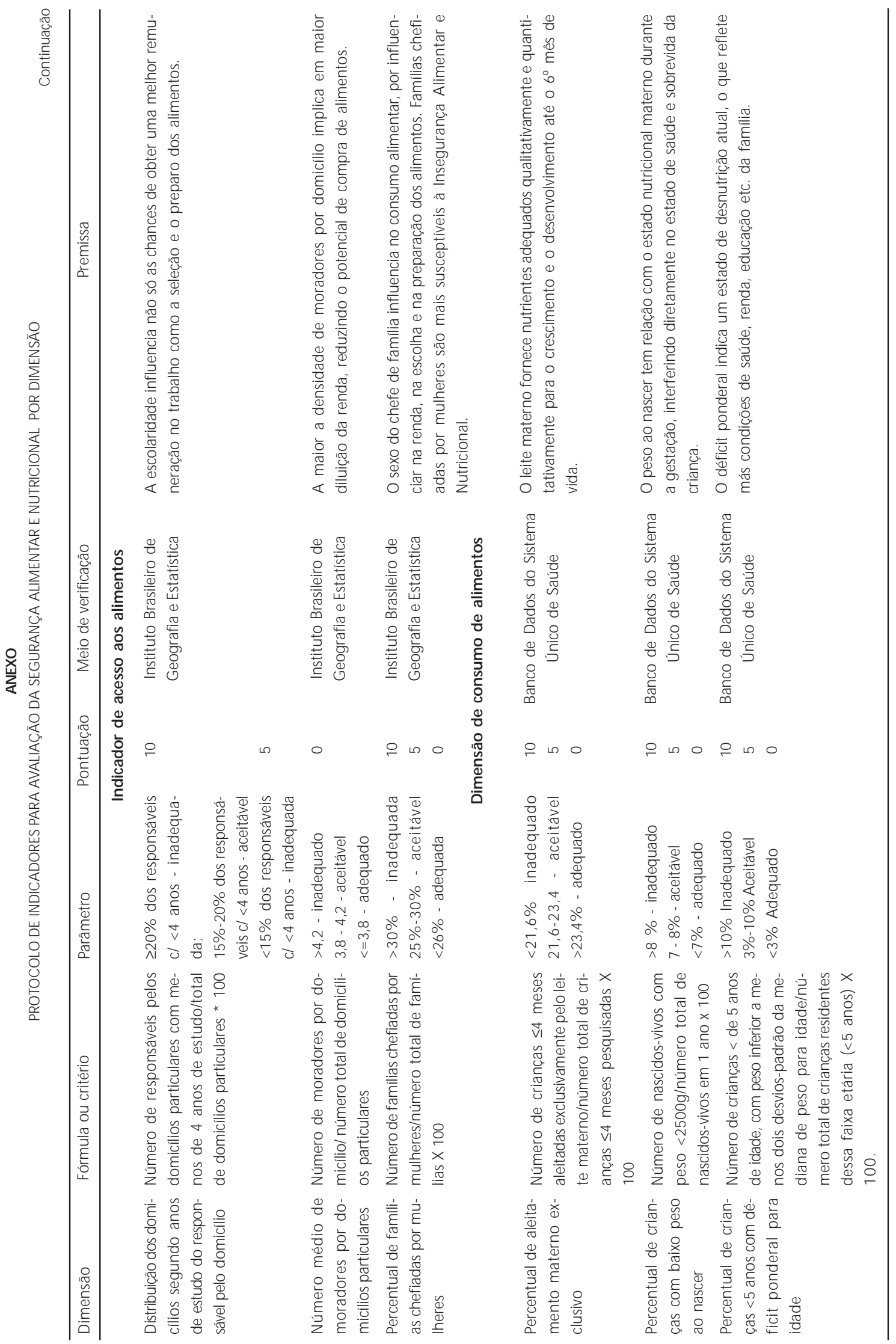




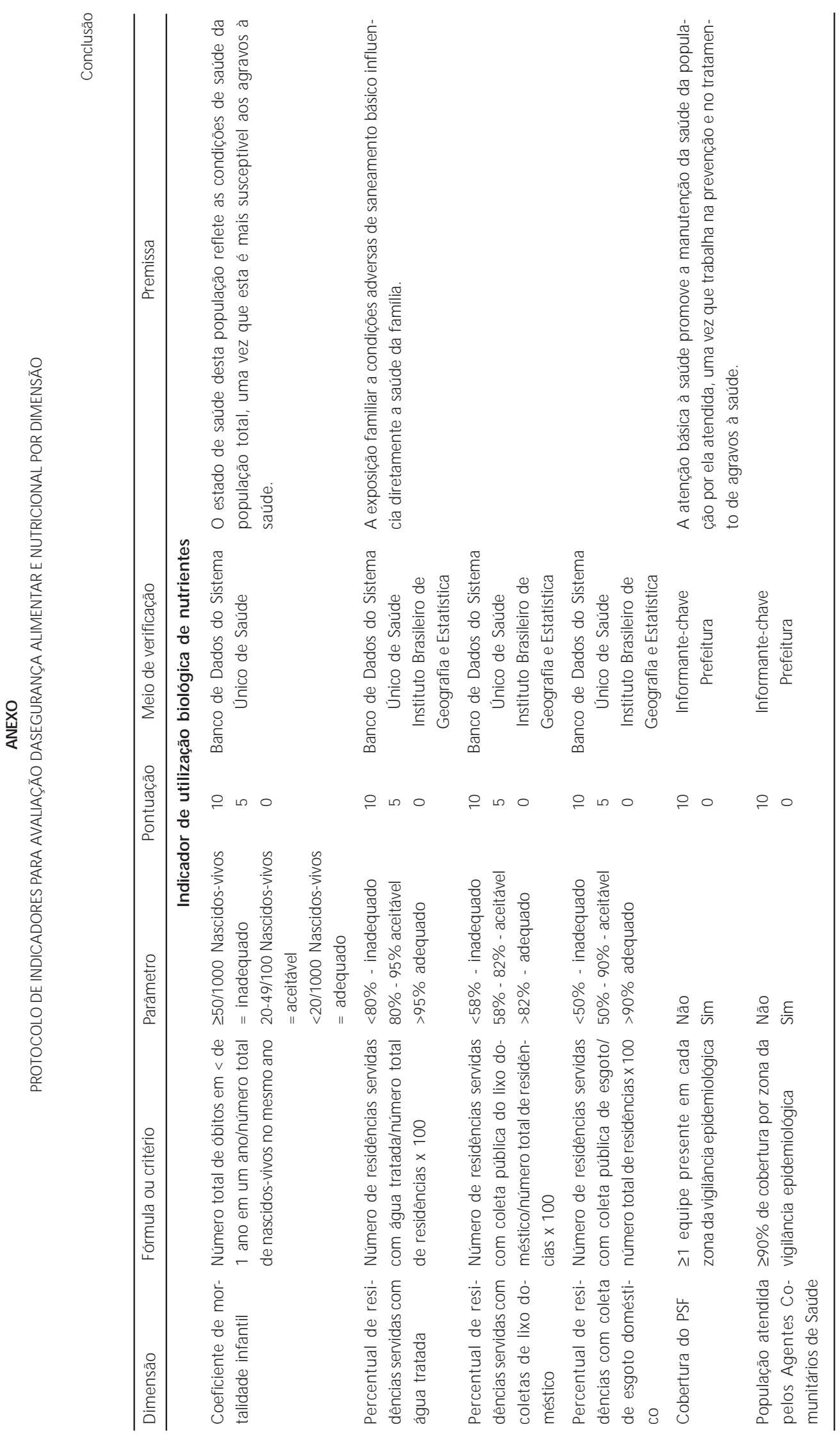


